\title{
Airborne dissemination of transmissible bacterial species in cystic fibrosis
}

\author{
Andrew M Jones
}

Delivery of multidisciplinary care by experienced teams in specialist units has conferred considerable benefits in care for individuals with cystic fibrosis (CF), with significant improvements in clinical outcome and survival figures. However, one particular concern of congregating patients at specialist CF centres is the potential for crossinfection. Over the past decade, the emergence and spread of clonal (transmissible) Pseudomonas aeruginosa strains among patients with $\mathrm{CF}$ has become an international problem. ${ }^{1-3}$ Case series suggest that initial infection with clonal $P$. aeruginosa strains may be more difficult to eradicate, ${ }^{4}$ and in cohort studies chronic infection is associated with worse clinical outcomes. ${ }^{156}$ The mechanism of spread of P. aeruginosa between patients with CF is unresolved. Potential routes include direct or indirect contact, droplet spread of relatively large infectious particles that travel short distances or airborne spread of smaller particles containing microorganisms that travel further and remain suspended for longer periods. It has been shown that patients with CF infected with clonal $P$. aeruginosa can contaminate their hands and their immediate environment for brief periods. ${ }^{7}$ In addition, clonal $P$. aeruginosa have been isolated from $\mathrm{CF}$ clinics by room air sampling. $^{78}$

Knibbs and coworkers present the findings of their recent work using equipment they developed with an aerobiological sampling system to measure the viability of $P$. aeruginosa in cough-generated aerosols from patients with $\mathrm{CF}^{9}$ Their studies

Correspondence to Dr Andrew M Jones, Consultant Physician and Honorary Reader, Manchester Adult Cystic Fibrosis Centre, University Hospitals South Manchester NHS Foundation Trust, Southmoor Road, Manchester, M23 9LT, UK; andrew.jones@uhsm.nhs.uk, andmarkj@hotmail.com demonstrate that after a $5 \mathrm{~min}$ period of intense coughing by a patient with $\mathrm{CF}$, viable $P$. aeruginosa can frequently be isolated from room air at a distance of at least $4 \mathrm{~m}$ from the source and can persist for $45 \mathrm{~min}$. The authors confirmed by bacterial fingerprinting techniques that the strains of $P$. aeruginosa cultured from the airborne particles corresponded to those harboured by the patients. Although the mechanism of cross-infection remains unknown, and ethical considerations limit the attainment of robust scientific proof, the findings of the study support the airborne route as one plausible means for transmission of $P$. aeruginosa infection between patients.

The present study may have implications for infection control practice and the design and functioning of CF centres. Previously infection control guidance has focused largely on avoidance of transmission by direct contact through measures such as hand hygiene, and droplet spread of relatively large infectious particles that travel short distances, with some guidelines suggesting people with CF maintain a distance of 1-3 $\mathrm{m}$ to reduce risk of infection. While precautions against contact spread of pathogens remain as essential as ever, future infection control policies will also need to allow greater consideration to potential airborne transmission of pathogens. It will be important to address the design and ventilation of buildings used for CF care and the administrative issues surrounding the use of these facilities. At large CF centres, there are often competing demands between the requirements to reuse rooms with providing sufficient time to allow dispersal of airborne contamination once a room has been vacated. Knibbs and colleagues modelled the influence of room ventilation on decay of airborne $P$. aeruginosa counts and provide data on the removal of viable airborne organisms with different air exchange rates. The concentration of viable organisms fell both with time and distance from the subject and, as expected, viable colony forming unit counts decreased quicker with greater air exchange rates, but even at 10 air exchanges per hour their modelling suggests that it was over $10 \mathrm{~min}$ before $90 \%$ of viable $P$. aeruginosa was removed.

Recommending exactly how long a room should be left vacant before it is used again is not straightforward. It is not possible to accurately predict any airborne infection risk due to several reasons, one of which is the lack of knowledge of the inoculum required to initiate infection, which may differ from strain to strain, and ethical concerns of performing relevant studies. There are also other factors: susceptibility to infection may differ from patient to patient; the environmental characteristics at any time such as humidity and temperature will influence the concentration and viability of airborne gramnegative pathogens; although air exchange rates can be calculated, in practice both the mixing of air within a room and contamination within a given area of the room are likely to be unequal. The present study by Knibbs and colleagues also provides evidence that there is a potentially large difference between individual patients in their ability to disseminate $P$. aeruginosa.

Administrative changes such as rotating staff rather than patients through clinic rooms, abolishing the need for waiting areas through careful timetabling of clinic slots and preventing congregating at other areas within the hospital (eg, direct dispensing of medications to patients within clinic rooms) may reduce risk of exposure to airborne pathogens. The applicability of such measures will depend on local resources and prevalence of infections. Other measures such as wearing of personnel protective equipment (face masks) by patients to protect themselves and other patients have been adopted at a number of CF centres in some countries. The requirement of wearing face masks and other potential strategies to reduce airborne infection, 
including ultraviolet filtration and mechanical room ventilation of facilities, is unresolved. An increasing use of telemedicine could provide a means of reducing need for travel to centres and thus reducing risk of cross-infection. The relative importance of individual components of infection control strategies is often difficult to evaluate as commonly several interventions are implemented consecutively in successful control of outbreaks. However, lessons learnt through ongoing epidemiological studies of cross-infection at CF centres will be important as they will help to guide consensus opinion of relevant infection control practice in CF. The subject of infection control practice is perhaps the most contentious issue in the CF community. Any recommendations that impose restraints within and outside of CF centres will be controversial. The exchange of views in response to the recent draft US CF Foundation recommendations highlight how opinions can be polarised in circumstances where direct evidence is commonly not available and is unlikely to be obtained due to ethical constraints. ${ }^{10} 11$

Although there was universal and often prolonged airborne dissemination of $P$. aeruginosa in the experimental model of Knibbs and colleagues, clonal $P$. aeruginosa strains have not have rapidly spread to infect all patients at CF centres. Factors that influence the capability of strains to transmit between patients are unknown, but it does not appear to be as simple as their ability to exist as viable organisms as airborne particles. Knibbs and colleagues studied 19 patients, 12 of whom were infected with clonal strains of $P$. aeruginosa. Both clonal and non-clonal strains were cultured from room air with no obvious difference in viability between different strains. Nevertheless, potential airborne transmission of $P$. aeruginosa infection still at this stage cannot be discounted. Spread of some respiratory pathogens, such as Mycobacterium tuberculosis and Influenza, is strongly linked to airborne transmission.
The accumulated experience of crossinfection in CF centres provides a cautionary message for the practice of congregating potentially susceptible patients who may harbour respiratory pathogens at specialist units, at a time when subspecialisation of respiratory services, such as the development of specialist non-CF bronchiectasis clinics, is becoming increasingly commonplace. A recent UK study did not find evidence of infection with dominant clones in unrelated patients at one specialist non-CF bronchiectasis clinic. ${ }^{12}$ However, clinical teams should be alert to any possibility of transmission of bacteria between patients with chronic respiratory conditions, through monitoring rates of infection with pathogens among patient cohorts and the use of microbiological strain typing in conjunction with epidemiological data to evaluate whether cross-infection has occurred.

In summary, the study by Knibbs and colleagues has provided additional data that support plausible transmission of $P$. aeruginosa between patients by an airborne route. It dispels the assumption of a 'safe' short (1-3 m) distance between patients. Although any actual risk from airborne transmission within CF centres remains difficult to quantify, it is a timely reminder for $\mathrm{CF}$ teams to work with their infection control colleagues and building design engineers to ensure patients can still benefit from provision of care from specialist and experienced multidisciplinary teams while minimising any potential risk from airborne organisms at CF centres.

\section{Competing interests None.}

Provenance and peer review Not commissioned; internally peer reviewed.

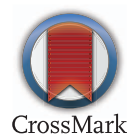

To cite Jones AM. Thorax 2014;69:690-691.

Received 5 May 2014

Revised 15 May 2014

Accepted 16 May 2014
Published Online First 4 June 2014

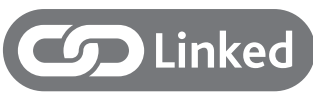

http://dx.doi.org/10.1136/thoraxjnl-2014-205213

Thorax 2014;69:690-691.

doi:10.1136/thoraxjnl-2014-205385

\section{REFERENCES}

1 Aaron SD, Vandemheen KL, Ramotar K, et al. Infection with transmissible strains of Pseudomonas aeruginosa and clinical outcomes in adults with cystic fibrosis. JAMA 2010;304:2145-53.

2 Kidd TJ, Ramsay KA, Hu H, et al. Shared Pseudomonas aeruginosa genotypes are common in Australian cystic fibrosis centres. Eur Respir J 2013;41:1091-100.

3 Jones AM, Govan JR, Doherty CJ, et al. Spread of a multiresistant strain of Pseudomonas aeruginosa in an adult cystic fibrosis clinic. Lancet 2001;358:557-8.

4 Gilchrist FJ, France M, Bright-Thomas R, et al. Can transmissible strains of Pseudomonas aeruginosa be successfully eradicated? Eur Respir J 2011;38:1483-6.

5 Al-Aloul M, Crawley J, Winstanley C, et al. Increased morbidity associated with chronic infection by an epidemic Pseudomonas aeruginosa strain in CF patients. Thorax 2004;59:334-6.

6 Jones AM, Dodd ME, Morris J, et al. Clinical outcome for cystic fibrosis patients infected with transmissible $P$. aeruginosa: an 8 year prospective study. Chest 2010;137:1405-9.

7 Panagea S, Winstanley C, Walshaw MJ, et al. Environmental contamination with an epidemic strain of Pseudomonas aeruginosa in a Liverpool cystic fibrosis centre, and study of its survival on dry surfaces. J Hosp Infect 2005;59:102-7.

8 Jones AM, Govan JR, Doherty CJ, et al. Identification of airborne dissemination of epidemic multiresistant strains of Pseudomonas aeruginosa at a CF centre during a cross infection outbreak. Thorax 2003;58:525-7.

9 Knibbs L, Johnson G, Kidd T, et al. Viability of Pseudomonas aeruginosa in cough aerosols generated by persons with cystic fibrosis. Thorax 2014;69:740-5.

10 Jain M, Saiman LM, Sabadosa K, et al. Point: Does the Risk of Cross-Infection Warrant Exclusion of Adults with Cystic Fibrosis from Cystic Fibrosis Foundation Events? Yes. Chest 2014;145:678-80.

11 Shepherd SL, Goodrich EJ, Desch J, et al. Counterpoint: does the risk of cross infection warrant exclusion of adults with cystic fibrosis from cystic fibrosis foundation events? No. Chest 2014;145:680-3.

12 De Soyza A, Perry A, Hall AJ, et al. Molecular epidemiological analysis suggests cross-infection with Pseudomonas aeruginosa is rare in non-cystic fibrosis bronchiectasis. Eur Respir J 2014;43:900-3. 